\title{
Acknowledgment of Principal and Ad Hoc Reviewers (2017)
}

\section{Principal Reviewers}

The Editor gratefully acknowledges the following persons who served as Principal reviewers for Personality Disorders: Theory, Research, and Treatment, reviewing a minimum of 3 manuscripts in the past year.

\begin{tabular}{|c|c|c|c|}
\hline Steve Balsis & Nicholas Eaton & John G. Kerns & Susan C. South \\
\hline $\begin{array}{l}\text { Daniel M. Blonigen } \\
\text { Marina A. Bornovalova }\end{array}$ & Janine D. Flory & Scott O. Lilienfeld & David R. Strong \\
\hline & Andrea Fossati & & Timothy J. Trull \\
\hline Keith Campbell & Josh Foster & Kristian E. Markon & \\
\hline Ryan William Carpenter & & Joshua D. Miller & David D. Vachon \\
\hline Allan D. Clifton & $\begin{array}{l}\text { Lauren Haliczer* } \\
\text { Michael Hallquist }\end{array}$ & Stephanie N. Mullins-Sweatt & Noah Venables \\
\hline $\begin{array}{l}\text { Matt DeLisi } \\
\text { Karen Derefinko } \\
\text { Hilary DeShong } \\
\text { Katherine L. Dixon-Gordon }\end{array}$ & $\begin{array}{l}\text { Brian M. Hicks } \\
\text { Christopher J. Hopwood }\end{array}$ & $\begin{array}{l}\text { Douglas B. Samuel } \\
\text { Martin Sellbom } \\
\text { Carla Sharp }\end{array}$ & $\begin{array}{l}\text { Amy W. Wagner } \\
\text { Glenn D. Walters } \\
\text { Aidan G. C. Wright } \\
\text { Dustin Wygant }\end{array}$ \\
\hline
\end{tabular}

\section{Ad Hoc Reviewers}

The Editor gratefully acknowledges the following persons who reviewed manuscripts submitted to Personality Disorders: Theory, Research, and Treatment in the past year. Reviewers who reviewed manuscripts under the supervision of a primary reviewer are denoted with an asterisk.

$\begin{array}{ll}\begin{array}{l}\text { Robert Ackerman } \\ \text { Saz Ahmed } \\ \text { Emily Ansell } \\ \text { Samuel Abplanalp* }\end{array} & \begin{array}{l}\text { Elizabeth Edershile* } \\ \text { Grant Edmonds }\end{array} \\ \text { Max Barranti } & \begin{array}{l}\text { Kostas Fanti } \\ \text { Julia Felton* } \\ \text { Sarah Fischer } \\ \text { Christopher Berghoff* }\end{array} \\ \text { Pavel S. Blagov } & \begin{array}{l}\text { Daniel Fulford } \\ \text { Christopher Funes* }\end{array} \\ \text { Michael Boudreaux } & \text { Andrea L. Glenn } \\ & \text { Kim L. Gratz } \\ \text { Nicole Cain } & \\ \text { Erica Carlson } & \text { Sarah Hampson } \\ \text { Kathy Carroll } & \text { Brooke M. Huibregtse } \\ \text { Alexander L. Chapman } & \text { Courtland S. Hyatt } \\ \text { David Chester } & \\ \text { John F. Clarkin } & \text { Roman Kotov } \\ \text { Alex S. Cohen } & \text { John Kurtz } \\ \text { Christopher C. Conway } & \\ \text { Lora Cope } & \text { Justin A. Lavner } \\ \text { Cristina Marie Crego } & \text { Holly Laws } \\ \text { Sheila E. Crowell } & \text { Carl W. Lejuez } \\ \text { Mark Dadds } & \text { Greg Lengel } \\ \text { Barbara De Clercq } & \text { Mark F. Lenzenweger } \\ \text { Colin DeYoung } & \text { Donald R. Lynam } \\ \text { Laura E. Drislane } & \\ & \text { Jessica F. Magidson } \\ & \text { Jessica L. Maples-Keller }\end{array}$

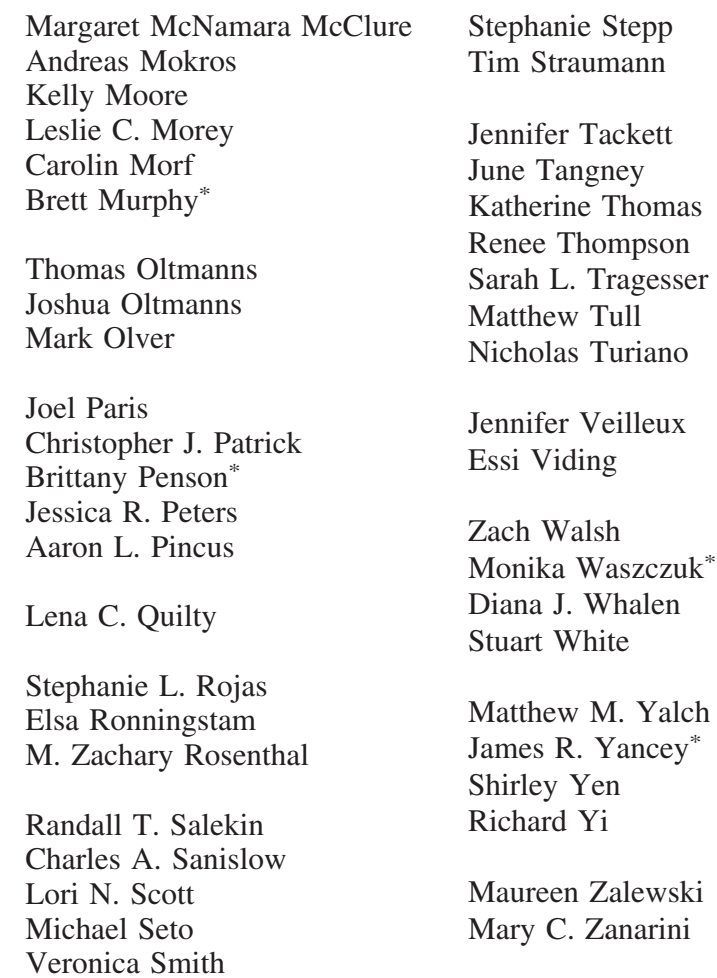

